\title{
ACCURACY IN COLOR MATCHING OF INCANDESCENT LIGHT SOURCES
}

\author{
BY \\ W. E. FORSYTHE
}

The relative distribution of energy in the visible spectrum of various radiating bodies for different temperatures has long been a matter of interest. There are two methods for obtaining directly such relative distributions in the visible spectrum, the one is by using some form of a spectrophotometer where the comparisons are made by eye observations, the other by using one of the methods where the energy is compared by means of some sort of an energy measuring device.

Owing to the decreasing sensibility of the eye, observations with the spectrophotometer are very difficult at the extreme red or blue end of the spectrum. It is also very difficult to make accurate measurements with a bolometer or thermopile in the extreme blue end of the spectrum.

There is a third method of obtaining such relative distributions that has some advantages over the two mentioned above. This method consists in matching in color the light from the source studied with that from a black body or other standard source. Such comparisons can be very accurately made with the ordinary Lummer-Brodhun contrast photometer. To do this the source studied is mounted on one side of the photometer and a comparison lamp on the other side. Suppose that at the start the comparison lamp is too low; then when there is a brightness match the trapezoid that is illuminated by the comparison lamp will appear reddish as compared with the other, which appears bluish. If now the voltage applied to the comparison lamp is raised a small amount and at the same time the photometer moved so that there is an intensity match, as observed in the photometer, the comparison trapezoid will, if the change has not been too great, appear less reddish than before. By repeating this process the comparison 
lamp can be very accurately matched in color with the source being studied. The source being studied is now to be removed and replaced with the standard lamp and the process repeated just as before excepting that in this case the voltage applied to the standard lamp is to be varied and the comparison kept constant at the voltage above obtained. Thus the standard source can be brought to a color match with the comparison source. In this way the source studied and the standard lamp are color matched by the substitution method. It has been found experimentally that a black body and most radiating solids can thus be color matched. Also most of the flames that have been used as light sources can be color matched with the black body. However, the Welsbach gas lamp and the sources that do not have a continuous spectrum cannot be so compared. For any source that can be color matched with the standard, the distribution of energy in the visible spectrum can be obtained much more accurately and with much less work than by either of the other methods, as will be shown below.

Color matching by different observers. In order to see how different observers would agree in their setting, two lamps were color matched by the substitution method by a number of observers. All the observers except one made three sets of five readings each on the voltage of the two lamps for color match with a third lamp of the same type held at a constant voltage.

In Table 1 are given the results of this test. The first three observers as listed in the table had had considerable experience with this kind of work. The fourth observer had had some previous experience while the last two had had no previous experience. The color temperature of the lamps used in this and the following test was about $2400^{\circ} \mathrm{K}$. The illumination on the photometer screen for this test was 5.1 foot candles. The averages of the voltages obtained in each set by each observer for lamp No. 4 to color match lamp No. 5 at 105 volts are given. There is also given the mean variation in volts for the different readings for the different observers. An examination of the table will show that the maximum range in the three sets for any of the experienced observers is less than 0.4 volt. The range in the 
TABLE 1

Values obtained for color matching lamp No. 5 against lamp No. 4 by a number of observers

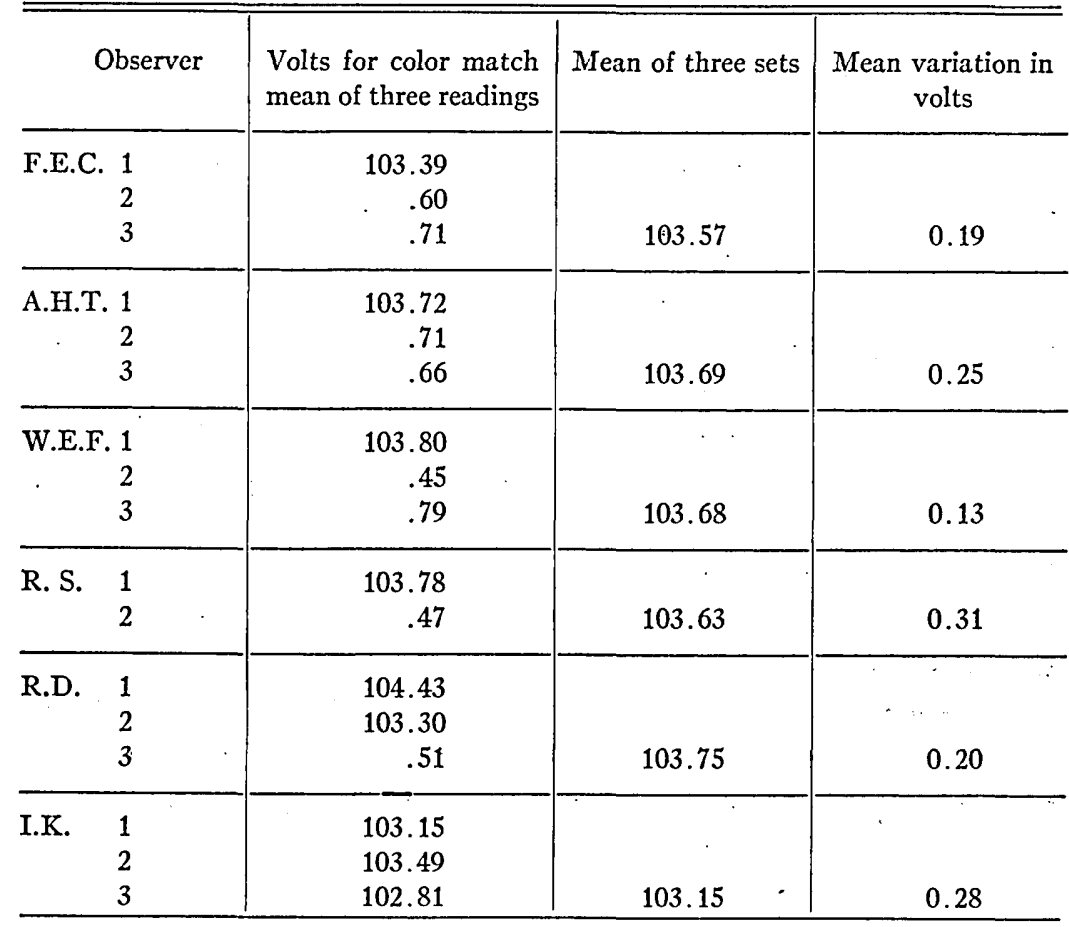

averages of the three sets for these same observers is only about one tenth volt. The maximum range in the averages for all the observers is only about five tenths volt. A change of one volt at this point corresponds to a change in color temperature of about $8^{\circ} \mathrm{K}$. It can thus be seen that the maximum range for inexperienced observers corresponds to about $4^{\circ} \mathrm{K}$. A range of $4^{\circ} \mathrm{K}$ at this temperature corresponds to an error of about $0.6 \%$ in relative energy between the red $(\lambda=.665 \mu)$ and the blue $(\lambda=.467 \mu)$. This is much better than the accuracy generally claimed in spectral distribution work.

The photometer used in making the color matches is of the Lummer-Brodhun contrast type having a contrast of about eight per cent. The reason for using this type is because it has been 
found to be much easier to make color matches with a contrast photometer. However, such matches can be made with an ordinary equality of brightness photometer. To see if any differences would be obtained several sets were made with a photometer having a contrast of about three and one half per cent. Although it seemed much harder to make color matches with this photometer, the final results were in very good agreement with those obtained with the other photometer.

Color matching at different intensities of illumination on the photometer screen. A test was also made to find out what accuracy could be expected in such work for different intensities of illumination on the photometer screen. In this test, different observers made quite a number of color matches for different illuminations on the photometer screen using in every case two tungsten lamps so that there would be no question as to the exactness of color match. The range of illumination on the photometer screen was from $1.8 \mathrm{ft}$. candles to $45 \mathrm{ft}$. candles.

Since it would require too much time and be too great a strain on the eyes for an observer to make readings at each of the intensities of illuminations at one time the following method was adopted. A set of readings was made each time at an illumination of $2.8 \mathrm{ft}$. candles taken as the standard and at one other illumination. In this way, the readings for each of the illuminations can be compared with the standard illumination. Two complete sets of readings were made at each illumination by each observer. In the first set, five readings were made at the standard illumination and then five at one of the other illuminations. In the second set of readings, three sets of three readings each were made at the standard illumination and alternating with these two sets were made at one of the other illuminations. The mean variations in volts in the readings on lamp No. 5 for color match with a standard lamp operated at a constant voltage for the different illuminations are given in Table 2. The variations for the standard illumination is given along with the variations for the other illuminations taken in the same set. The observers in the test with one change were the same as those used in the first test. The additional observer had had no previous experience. 
If there is any difference in the accuracy depending upon the illuminations, it is not very definitely shown by the data. There are slight indications that observer No. 1 sets better at low illumination and observer No. 3 better at high illumination. However, the difference is but little more than their error in setting.

The average variations of the entire group is given for the different illuminations and they show no very definite advantage for either the high or the low illumination. As before, one volt change corresponds to a change of about $8^{\circ} \mathrm{K}$, color temperature.

TABLE 2

Mean variation in readings in volts for color matching at different intensities by a number of observers

\begin{tabular}{|c|c|c|c|c|c|c|c|c|c|c|}
\hline \multirow{2}{*}{$\begin{array}{l}\text { Set............. } \\
\text { Illumination in } \\
\text { ft. candles ...... }\end{array}$} & \multicolumn{2}{|c|}{ I } & \multicolumn{2}{|c|}{ II } & \multicolumn{2}{|c|}{ III } & \multicolumn{2}{|c|}{ IV } & \multicolumn{2}{|c|}{ V } \\
\hline & 2.8 & 45.1 & 2.8 & 25.8 & 2.8 & 11.3 & 2.8 & 6.3 & 2.8 & 1.8 \\
\hline Observers & & & Mean & variati & $\mathrm{s}$ in & Its in & readir & & & \\
\hline F.E.C. & .16 & .22 & .17 & .20 & .10 & .21 & .22 & .09 & .18 & .13 \\
\hline A.H.T. & .22 & .21 & .24 & .19 & .15 & .24 & .28 & .12 & .17 & .15 \\
\hline W.E.F. . & .21 & .15 & .20 & .13 & .21 & .16 & .18 & .22 & .24 & .14 \\
\hline R.H.S... & .22 & .11 & .32 & .23 & .42 & .21 & .22 & .13 & .15 & .21 \\
\hline L.B..... & .22 & .10 & .26 & .15 & .29 & .18 & .25 & .27 & 25 & .23 \\
\hline R. D.... & .20 & .12 & .16 & .24 & .23 & .26 & .17 & .30 & 10 & .20 \\
\hline $\begin{array}{l}\text { Average mean } \\
\text { variation at each } \\
\text { illumination.... }\end{array}$ & & .15 & & .19 & & .21 & & .19 & 22 & .18 \\
\hline
\end{tabular}

From the above results it can be seen that very good agreement can be obtained in making color matches by different observers. There does not seem to be any great advantage in having a high illumination on the photometer screen. Ordinarily in this laboratory in making color matches an illumination of from four to eight foot candles on the photometer screen is used. As was mentioned above the Lummer-Brodhun contrast photometer having a contrast of about eight per cent is used because it seems easier to make color matches with that contrast than with a contrast of about three and one half per cent. It is our intention to test this matter out using different degrees of contrast both above and below this amount. 
The color temperature scale above $2650^{\circ} \mathrm{K}$ used in this laboratory depends on readings made with an optical pyrometer having first a red and then a blue screen in the eyepiece on a black body. The black body was the one designed by Dr. Worthing ${ }^{1}$ of this laboratory and consisted of a small tungsten tube with small radial holes for the purpose of observation.

The temperature of the black body was determined from the reading when the red glass was used as a screen and the ratio of the red to the blue brightness gave the color temperature scale. To explain this, suppose that at a temperature of $2800^{\circ} \mathrm{K}$ the readings on the black body gave a ratio of red to blue brightness, on an arbitrary scale, of 0.6. Now if at some unknown temperature tungsten was found to give the same red to blue brightness it would be said to have a color temperature of $2800^{\circ} \mathrm{K}$. By taking a number of such readings both on the black body and on tungsten a color temperature scale for tungsten was determined. This method of determining color temperature together with the accuracy that can be obtained will be more fully discussed in a paper now in the process of preparation.

At the Bureau of Standards the color temperature scale in this region depends upon radiometric measurements made by Dr. Coblentz in the visible spectrum of a particular 500 watt gas-filled tungsten lamp. ${ }^{2}$ Mr. Priest, by using the rotary dispersion of quartz, has extended the scale above the temperature corresponding to the energy distribution found by Dr. Coblentz.

Comparison of color scale of Nela Research Laboratories with that of the Bureau of Standards. Two tungsten lamps were color matched against the color standards of this laboratory and then sent to the Bureau of Standards, where they were color matched against their color standards by Mr. Priest. One of the lamps was then returned to this laboratory where it was again color matched. The Bureau of Standards also sent a high efficiency lamp to this laboratory for a like check. The results are given in Table 3.

${ }^{1}$ A. G. Worthing, Phy. Rev. N. S. 10, 377; 1917.

${ }^{2}$ J. O. S. A., \& R. S. I., 6, pp. 30-34; Jan., 1922. 
TABLE 3

Intercomparison of Color Scales

\begin{tabular}{|c|c|c|c|c|}
\hline \multirow{2}{*}{ LAMP } & \multicolumn{4}{|c|}{ Color Temperature degrees Kelvin } \\
\hline & Nela & Bureau of Standards & Nela & Bureau of Standards \\
\hline $\begin{array}{l}900 \text { watt movie } \\
500 \text { watt gas-filled }\end{array}$ & 3091 & 3085 & $\begin{array}{l}3083 \\
2848\end{array}$ & 2848 \\
\hline
\end{tabular}

The good agreement obtained in the two laboratories (although the actual figures obtained were probably accidental) by two different methods in two different laboratories shows the agreement possible in such work.

\section{SUMMARY}

Two tests have been made of accuracy that can be obtained in color matching. The first test was for different observers. It was found that experienced observers agreed in their values for color match to about $3^{\circ} \mathrm{K}$ for a color temperature of about $2400^{\circ} \mathrm{K}$. The second test was for color matching for different illuminations on the photometer screen. No very great difference was obtained in the accuracy for a range in illumination from 1.8 foot candles to 45 foot candles.

The color temperature scale for a temperature of about $2900^{\circ} \mathrm{K}$ of this Laboratory was compared with that of the Bureau of Standards and a very good agreement found.

Nela Research Liboratories,

Cleveland, Ohio,

MARCH, 1922 